\section{BRAZIULIAN JOURNAL \\ OF MEDICAL AND BIOLOGICAL RESF.ARCH}

www.bjournal.com.br
ISSN 0100-879X

Volume 42 (10) 870-992 October 2009

BIOMEDICAL SCIENCES

AND

CLINICAL INVESTIGATION

Braz J Med Biol Res, October 2009, Volume 42(10) 979-987

Prevalence of vascular-endothelial growth factor, matrix metalloproteinases and tissue inhibitors of metalloproteinases in primary breast cancer

F.C. Jobim, N.L. Xavier, D.M. Uchoa, D.B. Cruz, M. Saciloto, N. Chemello and G. Schwartsmann

The Brazilian Journal of Medical and Biological Research is partially financed by
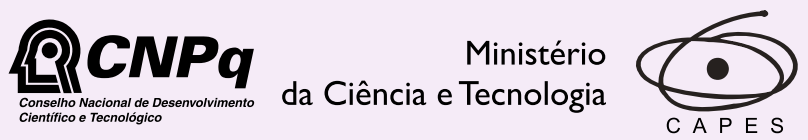

Ministério da Educação

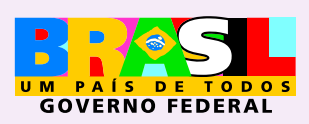

Institutional Sponsors 


\title{
Prevalence of vascular-endothelial growth factor, matrix metalloproteinases and tissue inhibitors of metalloproteinases in primary breast cancer
}

\author{
F.C. Jobim ${ }^{1,4}$, N.L. Xavier ${ }^{2}$, D.M. Uchoa ${ }^{3}$, D.B. Cruz ${ }^{3}$, \\ M. Saciloto ${ }^{4}$, N. Chemello ${ }^{2}$ and G. Schwartsmann ${ }^{1}$ \\ ${ }^{1}$ Programa de Pós-graduação em Medicina: Ciências Médicas, \\ ${ }^{2}$ Serviço de Mastologia, ${ }^{3}$ Serviço de Patologia, Hospital de Clínicas, \\ Universidade Federal do Rio Grande do Sul, Porto Alegre, RS, Brasil \\ ${ }^{4}$ Hospital Universitário, Universidade Federal de Santa Maria, \\ Santa Maria, RS, Brasil
}

\begin{abstract}
Our objective was to determine the presence of vascular endothelial growth factor (VEGF), matrix metalloproteinase-2 (MMP2) and MMP-9 and specific tissue inhibitors of matrix metalloproteinase (TIMP-1 and TIMP-2) in tumor samples obtained from patients with primary breast cancer. We attempted to correlate these findings with the status of the sentinel lymph node (SLN) and clinical-pathological characteristics such as age, tumor size, histological type, histological grade, and vascular invasion. Tumor samples from 88 patients with primary breast cancer were analyzed. The immunoreactivity of VEGF, MMP-2, MMP-9, TIMP-1, and TIMP-2 in tumors was correlated with clinical and pathological features, as well as SLN status. Nonparametric, Mann-Whittney, Kruskal-Wallis, and Spearmann tests were used. Categorical variables were analyzed by the Pearson test. No statistically significant correlation was found between the amount of VEGF, MMP-2, MMP-9, TIMP-1, and TIMP-2 and the presence of tumor cells in the SLN. However, larger tumor diameter $(P<0.01)$ and the presence of vascular invasion $(P<$ $0.01)$ were correlated positively with a positive SLN. A significant correlation of higher VEGF levels $(P=0.04)$ and lower TIMP-1 levels $(P=0.04)$ with ductal histology was also observed. Furthermore, lower TIMP-2 levels showed a statistically significant correlation with younger age ( $<50$ years) and larger tumor diameter $(2.0-5.0 \mathrm{~cm})$. A positive SLN correlated significantly with a larger tumor diameter and the presence of vascular invasion. Higher VEGF and lower TIMP-1 levels were observed in patients with ductal tumors, while higher TIMP-1 levels were observed in lobular tumors.
\end{abstract}

Key words: Breast cancer; Vascular endothelial growth factor; Matrix metalloproteinase; Metalloproteinase tissue inhibitors; Sentinel lymph node

\section{Introduction}

Pioneering work done by Judah Folkman in the 1970's suggested that tumor angiogenesis was a critical event for cancer growth (1). The interaction between tumor cells and stroma is a vital condition for the beginning of this process, involving molecules that include growth factors, adhesion molecules, angiogenic stimuli, clotting factors, and proteinases (2-4).

Some angiogenic proteins have been described. These include vascular endothelial growth factor (VEGF), basic fibroblast growth factor, tumor necrosis factor alpha, interleukin 8, and matrix metalloproteinases (MMPs) (5).
Endogenous angiogenesis inhibitors such as tissue inhibitors of matrix metalloproteinases (TIMPs) are necessary to block the mitogenic stimuli in the vascular endothelium $(5,6)$. Modifications in the expression of tumor angiogenic factors as well as their inhibitors have been described in various types of cancer, such as colorectal, lung, head and neck and breast cancer, and malignant melanoma $(7,8)$.

VEGF belongs to a family of glycoproteins that includes VEGF-A, VEGF-B, VEGF-C, VEGF-D, and placental growth factor. The main mediator of tumor angiogenesis is VEGFA, commonly referred to as VEGF $(2,8,9)$. A potent and

Correspondence: G. Schwartsmann, Hospital de Clínicas, Universidade Federal do Rio Grande do Sul, Rua Ramiro Barcelos, 2350/399, 90035-903 Porto Alegre, RS, Brasil. E-mail: gschwartsmann@hcpa.ufrgs.br

Research supported by the Postgraduate Program on Medical Science of the Federal University of Rio Grande do Sul and by Fundo de Incentivo à Pesquisa e Eventos, Academic Hospital of Porto Alegre (FIPE-HCPA).

Received August 10, 2008. Accepted June 22, 2009. Available online September 4, 2009. 
selective mitogenic factor for the endothelium, it elicits a rapid and complete angiogenic response and increased capillary permeability. Produced and secreted by a number of normal cells, it shows marked expression in tumor cells, including breast cancer $(10,11)$.

Several factors contribute to the increased expression of VEGF in breast cancer. Alow intracellular oxygen concentration blocks the degradation of hypoxia-inducing factor-1a, raising its levels and determining intracellular hypoxia, which in turn determines the increase in angiogenic activity for the activation of gene transcription of VEGF $(2,12,13)$. The cleavage of proteins by extracellular MMPs also frees VEGF and endostatin by the cleavage of collagen IV, the latter being an inhibitor of angiogenesis $(6,8)$.

The expansion of solid tumors determines the formation of hypoxic regions, leading to increased production of VEGF, which, in addition to determining the growth and survival of endothelial cells, also acts on some tumor cells, protecting them from apoptosis $(10,14,15)$.

The MMPs are neutral endopeptidases, the family of zinc-dependent proteinases. They play an important role in physiological processes, keeping a balance with the TIMPs. They are also involved in pathological conditions where there is a disruption of this balance, causing diseases such as arthritis, diabetic retinopathy, psoriasis, and cancer $(5,7)$.

Several studies suggest a significant involvement of the MMPs in the process of tumor growth and invasion. A high expression of MMPs, especially MMP-2 and -9 , is associated with the histological grade, stage of breast cancer and with the risk of recurrence and death $(3,5,7,16,17)$.

The MMPs are produced by a wide variety of connective tissue cells and also by a variety of malignant cells. Most are in latent forms (zymogens) that are cleaved proteolytically and activated in the extracellular space $(5,18)$.

The MMPs degrade the basement membrane and most components of the extracellular matrix, such as collagen, laminin, fibronectin, and elastin, which are barriers to the normal migration of cells and vascular invasion $(6,18)$.

Molecules with stromal action such as cyclooxygenase-2, adhesion molecules, MMPs, and VEGF accelerate the process of angiogenesis. However, for metastasis to occur, there must be a break-up of the basement membrane, degradation of the extracellular matrix, proliferation and migration of endothelial cells, formation of the vascular lumen, and functional maturation $(2,18)$.

Factors like age, tumor diameter, number of positive axillary lymph nodes, and hormonal and human epidermal growth factor receptor 2 (HER-2) status are currently used in breast cancer to guide the selection of the appropriate adjuvant therapy for women with locoregional disease (19). However, treatment decisions in patients with clinically negative axillary lymph nodes represent a special therapeutic challenge.

Immunohistochemistry has been used in an attempt to identify tumors with expression of certain molecules that may serve as biological target for new drugs, and can be combated by more specific and effective therapeutic regimens.

For the reasons given above, we determined by immunohistochemistry the expression of angiogenic factors (VEGF, MMP-2 and MMP-9) as well as TIMP-1 and TIMP2 in tumor samples obtained from patients with primary breast cancer.

Our objective was to correlate these findings with the status of the sentinel lymph node (SLN) and clinical-pathological characteristics such as age, tumor size, histological type, histological grade, and vascular invasion.

\section{Patients and Methods}

Ninety-five patients with a diagnosis of breast cancer, with clinically negative axillary lymph nodes and without distant metastases, were operated at the Academic Hospital in Porto Alegre (HCPA), Federal University of Rio Grande do Sul (UFRGS), between January and December 2004. Tissue samples obtained from the primary tumor and from the SLN were analyzed. This transverse study analyzed the expression of VEGF, MMP-2, MMP-9, TIMP-1, and TIMP2 in formalin-fixed and paraffin blocks with breast tumor samples. Hematoxylin-eosin preparations were checked and a paraffin block from each case was separated for immunohistochemical studies. Two cases of in situ carcinoma were excluded from the series, and five cases were discarded due to insufficient material, three of them showing a positive SLN for the presence of malignancy. Thus, 88 patients were included in the final analysis.

The clinical and pathological features were classified according to the following parameters: 1$)$ presence $(+)$ or absence (-) of tumor involvement in the SLN. The SLN was considered to be positive when it presented a cluster of malignant cells $\geq 0.2 \mathrm{~mm}$; ) age, $<50$ and $\geq 50$ years; 3 ) tumor diameter, categorized as $\leq 2.0 \mathrm{~cm}$ or between 2.0 and $\leq 5.0 \mathrm{~cm} ; 4$ ) histological types classified as ductal or lobular; 5) histological grade, classified as grade 1, grade 2, or grade 3, according to the Scarff Bloom Richardson score $(20) ; 6)$ presence of at least one nest of tumor cells clearly visible inside the vascular lumen (yes) or absence (no) of vascular invasion detected by hematoxylin-eosin.

The immunohistochemical technique using the standard avidin-biotin-peroxidase complex was employed to measure the expression of antigens in tumors. The immunoreaction was analyzed in a qualitative and quantitative way. The qualitative estimation was based on the staining intensity of the cytoplasm of neoplastic cells using the following scores: $0=$ absence of staining, 1 = slightly positive, 2 = moderately positive, 3 = strongly positive. Protein immunohistochemical expression was quantitated as percentage from 0 (zero) to $100 \%$. The final expression of each case was obtained by the following algorithm: HSCORE $=\Sigma[(I+1)] \times \mathrm{PC}$, where I 
and $P C$ represent the staining intensity and the percentage of stained cells, respectively $(21,22)$.

In order to measure the expression of VEGF we used the polyclonal antibody of rabbit antifactor of vascular human endothelial growth of Signet ${ }^{\mathrm{TM}}$, USA, and ultra streptavidin detection at 1:30 dilution, using a sample of breast tumor as external control. The expression of MMP-2 and MMP-9 was measured using the rat monoclonal antibody NCLMMP2-507, clone 17B11, and the rat monoclonal antibody NCL-MMP9-439, clone 15W2, from Novocastra Laboratories (UK) at 1:40 dilution, using normal hepatic tissue as the external control. The expression of TIMP-1 (tissue inhibitor of MMP-9) was analyzed with the rat monoclonal antibody NCL-TIMP1-485, clone 6F6a (Novocastra Laboratories) at 1:75 dilution, with normal colon tissue as the external control. To determine the expression of TIMP-2 (tissue inhibitor of MMP-2) the rat monoclonal antibody NCL-TIMP2, clone 3A4 (Novocastra Laboratories) was used at 1:25 dilution, with placental tissue as external control.

Three-micrometer thick paraffin sections were obtained, placed on histological slides treated with poly-D-lysine, deparaffinized with xylol, and rehydrated with $100 \%$ alcohol. The slides were washed in tap water and distilled water and incubated in PBS three times for 5 min each time to complete the blockade of endogenous peroxidase. Antigen retrieval was performed with citrate buffer, $\mathrm{pH} 6$, in a microwave oven for $20 \mathrm{~min}$ at $90^{\circ} \mathrm{C}$. After $15 \mathrm{~min}$ at room temperature, the slides were washed again in running and distilled water, and incubated in PBS. After application of $5 \%$ hydrogen peroxide, they were washed again and incubated in PBS. The sections were circled with a "DakoPen", the primary antibody was added, and the material was left in a moist chamber (Easy Path ${ }^{\circledR}$, Erviegas, Brazil) in the refrigerator overnight. The slides were then removed from the refrigerator and washed with distilled water. After receiving the secondary labeled streptavidin biotin (LSAB)
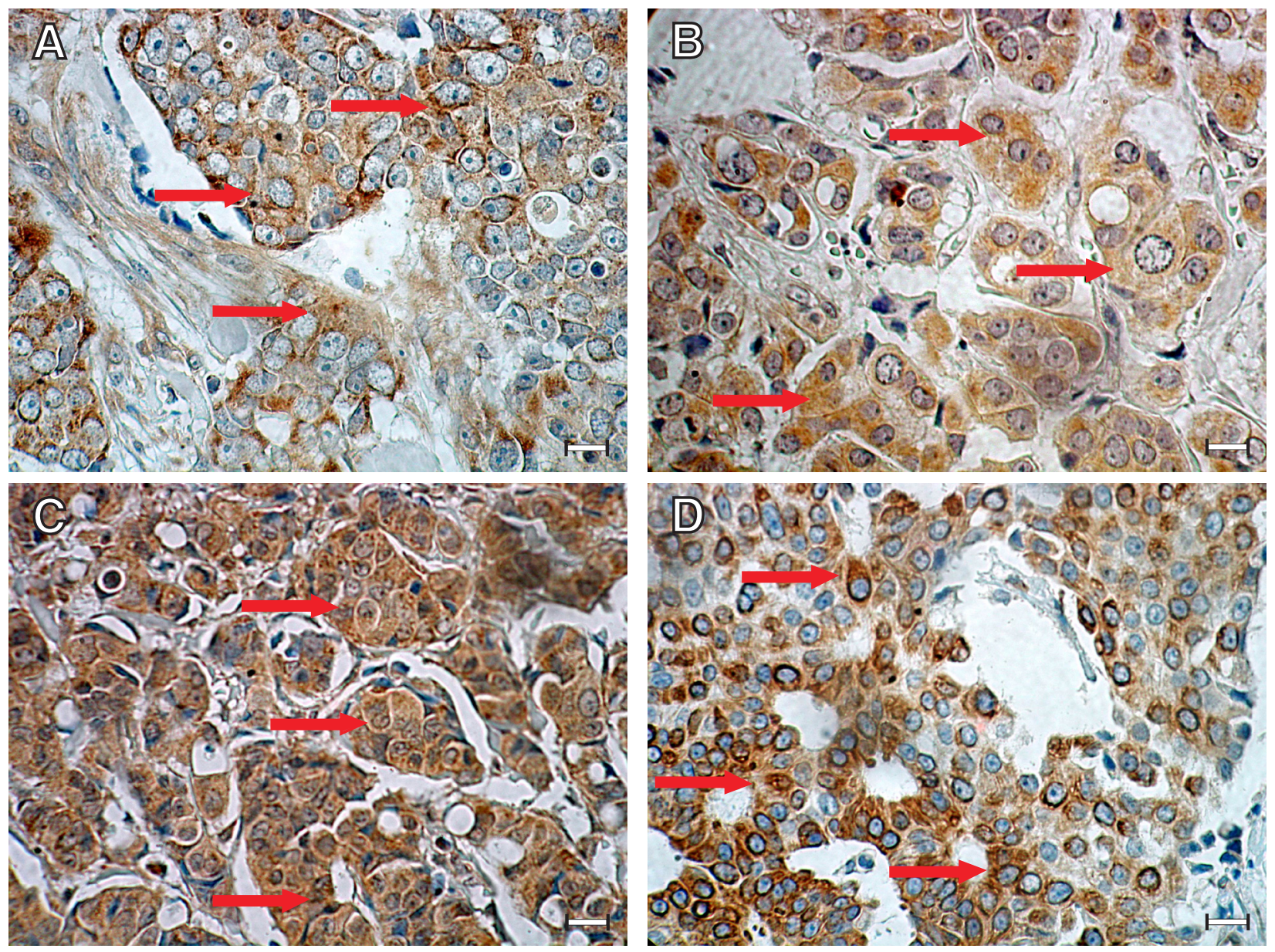

Figure 1. Immunohistochemical expression of matrix metalloproteinases MMP-2 (A), MMP-9 (B) and tissue inhibitors of metalloproteases [TIMP-2 (C) and TIMP-1 (D)] in neoplastic cells of a breast tumor. The arrows show the immunoreaction in the cytoplasm of the neoplastic cells. Breast cancer cells are shown at 400X magnification (bar $=20 \mu \mathrm{m}$ in all panels). 
antibody (Avidin Biotin, bottle 1, yellow, Dako, USA), the slides were placed in a moist chamber for $30 \mathrm{~min}$. After incubation in PBS, the procedure was repeated by adding the LSAB antibody (streptavidin peroxidase, Dako). The reaction was developed with the chromogenic substrate diaminobenzidine (Dako) for the sections to remain dark ( \pm $30 \mathrm{~s}$ ) and then were counter-stained with hematoxylin for 20-30 s. After rinsing under running water and application of $2 \%$ ammonia, the slides were rinsed in running water again, processed with $100 \%$ and mounted with xylol.

Semi-quantitative analysis was carried out by two independent pathologists in the Department of Pathology of HCPA, using an Olympus light microscope, UK, model BX-2 with 10 and $40 X$ objectives.

Qualitative immunohistochemical expression was considered to be positive when the tumor showed at least one cell with cytoplasmic clear and unambiguous brownish staining for each marker utilized, regardless of the intensity of coloration (Figure 1). The quantitative expression of each marker was taken as the average percentage of stained cells in at least 10 microscopic fields.

Statistical analysis to compare the association between SLN-positive groups versus SLN-negative groups and the factors under study and with the other variables selected was performed using the statistical program SPSS V15.
Continuous variables such as age and tumor diameter were compared by the Student $t$-test. VEGF, MMP-2, -9, TIMP-1, and -2 were correlated with the other clinical-pathological parameters by the non-parametric Mann-Whitney U-test, and the correlation with the histological grade was calculated by the non-parametric Kruskal-Wallis test. The Pearson chisquare test was used to analyze the categorical variables. The correlation of angiogenic factors with one another was calculated by the Spearman correlation coefficient. The confidence interval was set at $95 \%(95 \% \mathrm{Cl})$ and values of $\mathrm{P}<0.05$ were considered to be statistically significant.

The project was approved by the Postgraduate Research Group and Medical Ethics Commission of HCPA (\#01/88CNS).

\section{Results}

Table 1 shows the distribution of clinical-pathological and immunohistochemical data according to SLN (+) and SLN (-) status. Mean patient age of 88 patients was 59 years (range: $33-81$ years) and mean tumor diameter was $2.3 \mathrm{~cm}$ (range: $0.5-5.0 \mathrm{~cm}$ ). The most frequent histological type was infiltrating ductal carcinoma with 83 (94\%) cases, and infiltrating lobular carcinoma was present in 5 cases (6\%). The distribution of histological grade according to

Table 1. Clinical-pathological and immunohistochemical data in relation to sentinel lymph node status (SLN): positive and negative SLN groups.

\begin{tabular}{|c|c|c|c|}
\hline Variables & All $(N=88)$ & Positive SLN (N = 44) & Negative SLN $(N=44)$ \\
\hline Age $^{a}$ (years) & $58.7 \pm 11.3$ & $58.7 \pm 11.8$ & $58.8 \pm 11.0$ \\
\hline Tumor diameter ${ }^{a^{*}}(\mathrm{~cm})$ & $2.3 \pm 1.0$ & $2.7 \pm 1.1$ & $1.9 \pm 1.0$ \\
\hline \multicolumn{4}{|l|}{ Histological type ${ }^{\mathrm{b}}, \mathrm{N}(\%)$} \\
\hline Ductal & $83(94 \%)$ & $42(96 \%)$ & $41(93 \%)$ \\
\hline Lobular & $5(6 \%)$ & $2(5 \%)$ & $3(7 \%)$ \\
\hline \multicolumn{4}{|l|}{ Histological grade ${ }^{\mathrm{b}}, \mathrm{N}(\%)$} \\
\hline $\mathrm{G} 1$ & $23(26 \%)$ & $9(21 \%)$ & $14(32 \%)$ \\
\hline G2 & $53(60 \%)$ & $30(68 \%)$ & $23(52 \%)$ \\
\hline G3 & $12(14 \%)$ & $5.0(11 \%)$ & $7.0(16 \%)$ \\
\hline \multicolumn{4}{|c|}{ Vascular invasion ${ }^{\mathrm{b} *}, \mathrm{~N}(\%)$} \\
\hline Yes & $46(52 \%)$ & $37(84 \%)$ & $9(21 \%)$ \\
\hline No & $42(48 \%)$ & $7(16 \%)$ & $35(80 \%)$ \\
\hline VEGFC & $190(180-255)$ & $190(177-239)$ & $190(180-270)$ \\
\hline MMP-2 $^{\mathrm{c}}$ & $55(6-204)$ & $63(4-176)$ & $52(9-228)$ \\
\hline MMP-9c & $180(100-230)$ & $165(34-255)$ & $180(153-196)$ \\
\hline TIMP-1 ${ }^{\mathrm{C}}$ & $368(340-380)$ & $360(293-380)$ & $372(340-387)$ \\
\hline TIMP-2 ${ }^{\mathrm{C}}$ & $249(170-285)$ & $225(160-277)$ & $260(182-322)$ \\
\hline MMP-2/TIMP-2c & $0.30(0.03-0.81)$ & $0.34(0.01-0.77)$ & $0.26(0.05-0.88)$ \\
\hline MMP-9/TIMP-1c & $0.47(0.29-0.65)$ & $0.47(0.10-0.70)$ & $0.49(0.40-0.60)$ \\
\hline
\end{tabular}

Data are reported as means $\pm \mathrm{SD}$, score (percent), or median (interquartile range: $\mathrm{P} 25$ to $\mathrm{P} 75$ ). The expression of each protein measured by immunohistochemistry is reported as the mean of the algorithm: HSCORE $=\Sigma[(I+1)] \times P C$, where I and PC represent the staining intensity and the percent of stained cells, respectively. VEGF $=$ vascular endothelium growth factor; MMP $=$ matrix metalloproteinase; TIMP = tissue inhibitors of matrix metalloproteinase. ${ }^{*} \mathrm{P}<0.05$, significant correlation with positive SLN ( ${ }^{\mathrm{a}}$ t-test, ${ }^{\mathrm{b}}$ chi-square test, ${ }^{\mathrm{C}}$ Mann-Whitney U-test). 


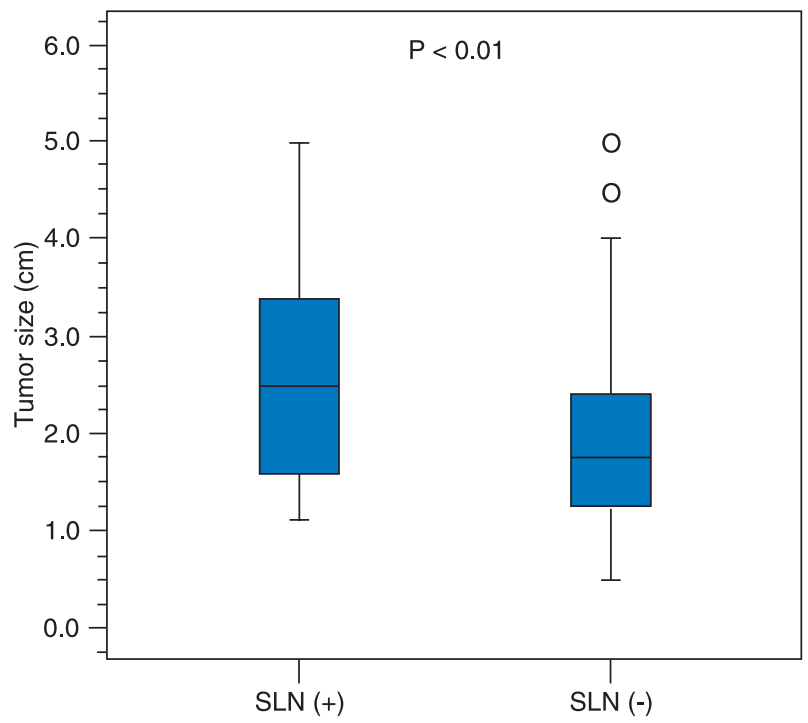

Figure 2. Correlation between tumor diameter and sentinel lymph node status (SLN): positive (+) and negative (-) SLN. P < 0.01 (Spearman).

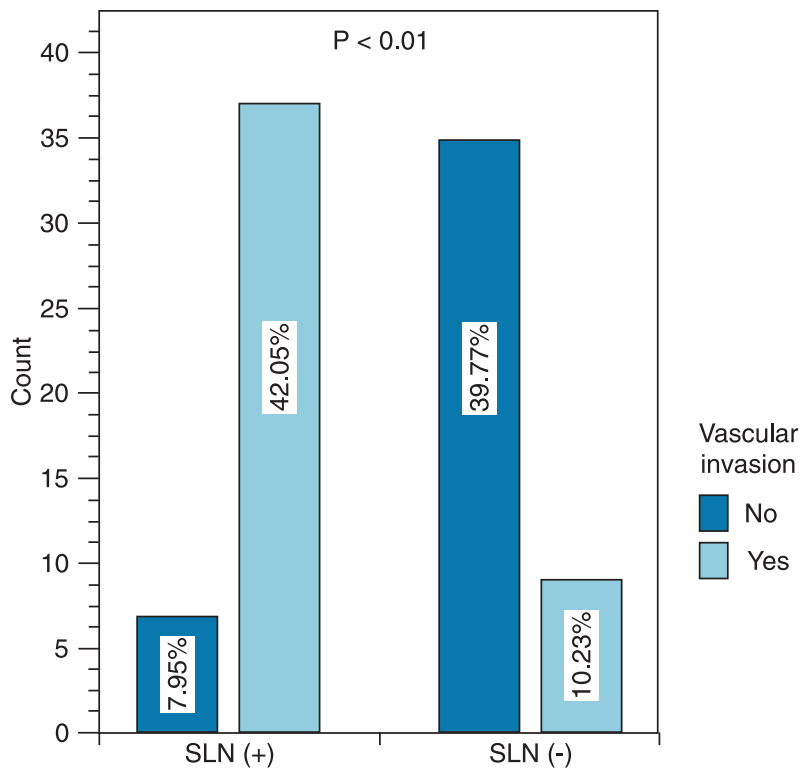

Figure 3. Correlation between the presence of vascular invasion and sentinel lymph node status (SLN): positive $(+)$ and negative (-) SLN. A positive correlation was observed between the presence of vascular invasion and the occurrence of a positive SLN; $\mathrm{P}<0.01$ (Spearman)

Table 2. Attempts to correlate the angiogenic factors VEGF, MMP-2 and MMP-9 with SLN, age, tumor diameter, histology, and vascular invasion.

\begin{tabular}{|c|c|c|c|c|}
\hline Variables & $\mathrm{N}(\%)$ & VEGF & MMP-2 & MMP-9 \\
\hline \multicolumn{5}{|l|}{ SLN } \\
\hline Positive & $44(50 \%)$ & $190(177-239)$ & $63(4.0-176)$ & $165(34-255)$ \\
\hline Negative & $44(50 \%)$ & $190(180-270)$ & $52(9.0-228)$ & $180(153-196)$ \\
\hline \multicolumn{5}{|l|}{ Age (years) } \\
\hline$<50$ & $21(24 \%)$ & $190(178-279)$ & $40(1.0-175)$ & $160(43-190)$ \\
\hline$\geq 50$ & $67(76 \%)$ & $190(180-225)$ & $80(3.0-190)$ & $180(120-255)$ \\
\hline \multicolumn{5}{|c|}{ Tumor diameter $(\mathrm{cm})$} \\
\hline$\leq 2$ & $47(53 \%)$ & $190(180-270)$ & $40(6.0-210)$ & $180(120-240)$ \\
\hline$>2$ and $\leq 5$ & $41(47 \%)$ & $190(176-248)$ & $75(7.0-210)$ & $170(53-207)$ \\
\hline \multicolumn{5}{|c|}{ Histological type } \\
\hline Ductal & $83(94 \%)$ & $190(180-264)^{*}$ & $54(6.0-210)$ & $180(100-219)$ \\
\hline Lobular & $5.0(6 \%)$ & $170(158-185)$ & $84(23-267)$ & $186(50-284)$ \\
\hline \multicolumn{5}{|c|}{ Histological grade } \\
\hline $\mathrm{G} 1$ & $23(26 \%)$ & $186(176-210)$ & $126(6.0-186)$ & $180(46-196)$ \\
\hline $\mathrm{G} 2$ & $53(60 \%)$ & $190(180-255)$ & $56(10-240)$ & $170(98-217)$ \\
\hline G3 & $12(14 \%)$ & $190(182-277)$ & $11(0.0-209)$ & $180(118-262)$ \\
\hline \multicolumn{5}{|c|}{ Vascular invasion } \\
\hline Yes & $46(52 \%)$ & $190(176-270)$ & $73(6.0-169)$ & $180(57-255)$ \\
\hline No & $42(48 \%)$ & $187(180-255)$ & $45(8.0-236)$ & $180(128-190)$ \\
\hline
\end{tabular}

Correlation coefficients were calculated by the Spearman correlation method. Data are reported as score (percent) or median (interquartile range: P25 to P75). The expression of each protein measured by immunohistochemistry is reported as the mean of the algorithm: HSCORE $=\Sigma[(I+1)] \times P C$, where I and PC represent the staining intensity and the percent of stained cells, respectively. SLN = sentinel lymph node; VEGF = vascular endothelial growth factor; MMP = matrix metalloproteinase. ${ }^{*} \mathrm{P}<0.05$, ductal compared to lobular histological type (Mann-Whitney U-test; Kruskal-Wallis H-test for histological grade). 
Table 3. Attempts to correlate the specific inhibitors TIMP-1, TIMP-2, MMP-2/TIMP-2 and MMP-9/TIMP-1 with SLN, age, tumor diameter, histology, and vascular invasion.

\begin{tabular}{|c|c|c|c|c|c|}
\hline Variables & $\mathrm{N}(\%)$ & TIMP-1 & TIMP-2 & MMP-2/TMP-2 & MMP-9/TIMP-1 \\
\hline \multicolumn{6}{|l|}{ SLN } \\
\hline Positive & $44(50 \%)$ & $360(293-380)$ & $225(160-277)$ & $0.34(0.01-0.77)$ & $0.47(0.10-0.70)$ \\
\hline Negative & $44(50 \%)$ & $372(340-387)$ & $260(182-322)$ & $0.26(0.05-0.88)$ & $0.49(0.40-0.60)$ \\
\hline \multicolumn{6}{|l|}{ Age (years) } \\
\hline$<50$ & $21(24 \%)$ & $360(330-370)$ & $178(149-237)$ & $0.29(0.01-0.95)$ & $0.47(0.11-0.58)$ \\
\hline$\geq 50$ & $67(76 \%)$ & $372(340-388)^{*}$ & $264(190-332)^{*}$ & $0.35(0.04-0.72)$ & $0.48(0.31-0.67)$ \\
\hline \multicolumn{6}{|c|}{ Tumor diameter $(\mathrm{cm})$} \\
\hline$\leq 2$ & 47 (53\%) & $372(340-380)$ & $270(190-291)^{*}$ & $0.18(0.03-0.70)$ & $0.48(0.31-0.67)$ \\
\hline$>2$ and $\leq 5$ & $41(47 \%)$ & $360(319-380)$ & $190(160-270)$ & $0.44(0.03-0.87)$ & $0.47(0.14-0.63)$ \\
\hline \multicolumn{6}{|c|}{ Histological type } \\
\hline Ductal & $83(94 \%)$ & $368(332-380)$ & $234(170-285)$ & $0.31(0.03-0.82)$ & $0.47(0.30-0.63)$ \\
\hline Lobular & $5(6 \%)$ & $388(360-394)^{*}$ & $270(255-366)$ & $0.22(0.11-0.82)$ & $0.48(0.14-0.72)$ \\
\hline \multicolumn{6}{|c|}{ Histological grade } \\
\hline G1 & $23(26 \%)$ & $380(340-388)$ & $225(160-285)$ & $0.57(0.03-0.82)$ & $0.50(0.12-0.57)$ \\
\hline G2 & $53(60 \%)$ & $368(340-380)$ & $249(170-306)$ & $0.30(0.07-0.83)$ & $0.47(0.28-0.66)$ \\
\hline G3 & $12(14 \%)$ & $350(293-386)$ & $245(200-281)$ & $0.04(0.00-0.60)$ & $0.50(0.33-0.72)$ \\
\hline \multicolumn{6}{|c|}{ Vascular invasion } \\
\hline Yes & $46(52 \%)$ & $360(296-380)$ & $230(160-230)$ & $0.32(0.02-0.79)$ & $0.49(0.15-0.73)$ \\
\hline No & $42(48 \%)$ & $372(340-381)$ & 255 (189-334) & $0.24(0.04-0.84)$ & $0.47(0.33-0.55)$ \\
\hline
\end{tabular}

Correlation coefficients were calculated by the Spearman correlation method. Data are reported as score (percent) or median (interquartile range: P25 to P75). The expression of each protein measured by immunohistochemistry is reported as the mean of the algorithm: HSCORE $=\Sigma[(I+1)] \times P C$, where I and PC represent the staining intensity and the percent of stained cells, respectively. SLN = sentinel lymph node; TIMP = tissue inhibitors of matrix metalloproteinase; MMP = matrix metalloproteinase. ${ }^{*} \mathrm{P}<0.05$ (Mann-Whitney U-test; Kruskal-Wallis $\mathrm{H}$-test for histological grade).

the Scarff Bloom Richardson score was classified as G1: $23(26 \%)$, G2: $53(60 \%)$ and G3: $12(14 \%)$. Vascular invasion was documented in $46(52 \%)$ cases. Tumor samples were analyzed in terms of the presence or absence of SLN involvement. Tumor diameter $(P<0.01$; Figure 2$)$ and the presence of vascular invasion in the primary tumor were correlated significantly $(P<0.01$; Figure 3 ) with a positive SLN.

Table 1 also shows the immunohistochemical expression of VEGF, MMP-2 and -9 and TIMP-1 and - 2 in the cytoplasm of neoplastic cells, measured by the means of HSCORE and the ratio between MMPs and their specific inhibitors (MMP-2/TIMP-2 and MMP-9/TIMP-1). VEGF presented a median score of 190 (180-255); MMP-2 presented a median score of 55 (6-204), with 14 (15.9\%) negative cases; MMP-9 presented a median score of 180 (100-230), with $10(11.4 \%)$ negative cases; TIMP-1 presented a median score of 368 (340-380), and TIMP-2 a median score of 249 (170-285). No significant correlation between the immunohistochemical expression of the proteins cited above and SLN status was observed.

Table 2 presents the immunoreactivity of VEGF, MMP-2 and MMP-9 in relation to the SLN status and other variables. Tumors with the lobular histological type showed a significantly lower VEGF than the ductal type $(P=0.04$; Figure 4).

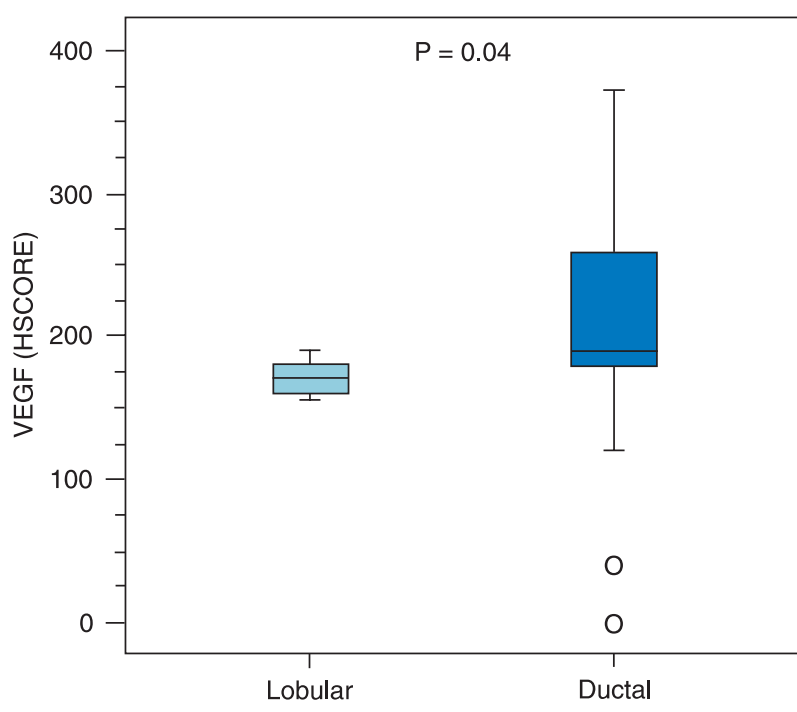

Figure 4. Correlation between vascular endothelial growth factor (VEGF) levels and histological tumor type. A positive correlation between VEGF expression and histological type was observed $(P=0.04 ;$ Spearman $)$.

In Table 3, SLN status and the variables cited above were correlated with TIMP-1 and TIMP-2 levels and with the ratio 
Table 4. Summary of statistically significant correlations between VEGF, MMP-2, MMP-9, TIMP-1, TIMP-2, MMP-2/ TIMP-2, and MMP-9/TIMP-1 levels identified by the Spearman correlation method.

\begin{tabular}{|c|c|c|c|c|c|c|c|}
\hline Factors & VEGF & MMP-2 & MMP-9 & TIMP-1 & TIMP-2 & MMP-2/TIMP-2 & MMP-9/TIMP-1 \\
\hline \multicolumn{8}{|l|}{ VEGF } \\
\hline rho & - & & 0.26 & & & & 0.32 \\
\hline $\mathrm{P}$ & - & & 0.01 & & & & 0.02 \\
\hline \multicolumn{8}{|l|}{ MMP-2 } \\
\hline rho & & - & & & 0.36 & 0.92 & \\
\hline $\mathrm{P}$ & & - & & & $<0.01$ & $<0.01$ & \\
\hline \multicolumn{8}{|l|}{ MMP-9 } \\
\hline rho & 0.26 & & - & & & & \\
\hline $\mathrm{P}$ & 0.01 & & - & & & & $<0.01$ \\
\hline \multicolumn{8}{|l|}{ TIMP-1 } \\
\hline rho & & & & - & & & \\
\hline $\mathrm{P}$ & & & & - & & & \\
\hline \multicolumn{8}{|l|}{ TIMP-2 } \\
\hline rho & & 0.36 & & & - & & \\
\hline $\mathrm{P}$ & & $<0.01$ & & & - & & \\
\hline \multicolumn{8}{|c|}{ MMP-2/TIMP-2 } \\
\hline rho & & 0.92 & & & & - & \\
\hline $\mathrm{P}$ & & $<0.01$ & & & & - & \\
\hline \multicolumn{8}{|c|}{ MMP-9/TIMP-1 } \\
\hline rho & 0.32 & & 0.95 & & & & - \\
\hline$P$ & 0.02 & & $<0.01$ & & & & - \\
\hline
\end{tabular}

SLN = sentinel lymph node; VEGF = vascular endothelium growth factor; MMP = matrix metalloproteinase; TIMP = tissue inhibitors of matrix metalloproteinase; rho $=$ Spearman rank correlation. Data are reported only for $\mathrm{P}<0.05$ (Spearman correlation coefficient).

between MMP-2 and MMP-9 and their specific inhibitors (MMP-2/TIMP-2 and MMP-9/TIMP-1). TIMP-1 levels were higher in tumors of the lobular type $(P=0.04)$ and TIMP-2 levels were higher in older patients $(P<0.01)$ and in smaller tumors $(P=0.01)$. The MMP-2/TIMP-2 and MMP-9/TIMP-1 ratios showed no significant correlation.

The correlation of VEGF, MMP-2, MMP-9, TIMP1, TIMP-2 levels with the MMP-2/TIMP-2 and MMP-9/ TIMP-2 ratios is shown in Table 4. VEGF levels correlated significantly with the MMP-9 factor $(P=0.01)$. A positive correlation was also observed between MMP-2 and TIMP-2 levels $(P<0.01)$.

\section{Discussion}

The recognition that the occurrence of tumor growth and metastasis depends on the angiogenesis process has promoted the investigation of prognostic and predictive factors such as hormone receptor, HER-2, VEGF and MMPs, in order to elucidate the significance of angiogenesis in breast cancer. The analysis of the expression of these biomarkers in tissues of breast cancer can be of great prognostic value in assessing the metastatic potential of tumor and survival of patients $(3,23)$

Several studies have suggested a relationship between VEGF, MMP-2, MMP-9, TIMP-1, and TIMP-2 expression in primary breast cancer and disease prognosis. Some of them suggested that patients with high levels of VEGF, MMP-2 and MMP-9 expression in the primary tumor might have a more aggressive clinical course, with presentation of early metastases. However, these results are still conflicting and not clear $(13,24,25)$.

Analyzing the literature, it was observed that the quantification of immunohistochemical expression of proteins in tumors is determined in different manners, using the most varied scores. All authors consider the presence of brown staining in neoplastic cells as a parameter for positivity. Some authors consider only the percentage of stained cells to set expression $(26,27)$, while most link the percentage of cells stained with the intensity of staining, but using various formulas (28-30). We applied the algorithm HSCORE, widely described in the literature $(19,20)$, in an attempt to use a method of compression and easy reproducibility.

In the present study, larger tumor diameter $(P<0.01)$ and the presence of vascular invasion $(P<0.01)$ were correlated significantly with the presence of a positive SLN. 
When comparing the expression of VEGF, MMP-2, MMP-9 and their specific inhibitors TIMP-1 and TIMP-2 to the presence or absence of tumor cells in the SLN, no statistically significant correlation was found.

Several authors, such as Nakopoulou et al. (31) and Pellikainen et al. (26), found high expressions of MMPs associated with small tumors (TI-2) and negative axillary lymph nodes. However, other authors, such as Kim et al. (32) and Hao et al. (30), found a significant association of VEGF and MMP-9 with advanced histological grade and positive axillary lymph node.

We found a significant correlation between higher VEGF expression $(P=0.04)$ and lower TIMP-1 levels $(P=0.04)$ with the ductal histological type. Furthermore, lower TIMP-2 levels also showed a significant correlation with younger age $(<50$ years $)(P=0.002)$ and a larger tumor diameter (2.0-5.0 cm; $P=0.01)$.

Nakopoulou et al. (33) studied 133 cases of invasive breast cancer and detected an increased TIMP-1 expression in $60 \%$ of cases, with an inverse correlation between TIMP-1 and histological grade $(P=0.003)$. The cases with increased TIMP-1 expression were correlated with favorable prognosis factors, mainly with negative axillary lymph nodes. Yet, elevated TIMP-1 levels were associated with better survival $(P=0.01)$.

Kim et al. (32), when analyzing 63 cases of T1-T2 breast tumors, did not find a correlation of TIMP-2 expression with histological grade or axillary lymph node status. A study of Têtu et al. (34) found the TIMP-2 expression associated with the ductal histological type.

In order to analyze the correlation of the balance between MMPs and their inhibitors with SLN and other variables under study, we calculated the MMP-2/TIMP-2 and MMP-9/TIMP-1 ratios. We did not find a significant correlation between these ratios and SLN involvement.

Jinga et al. (22) analyzed this ratio in benign and malignant breast tissues and found a significant difference in malignant tissues, suggesting that an abnormal balance between MMPs and TIMPs plays an important role in tumor growth patterns.

Analyzing the relationship MMP-2/TIMP-2, Têtu et al. (34) found a worse 5-year survival in those patients with low expression of TIMP-2 and high expression of MMP-2, but did not find an association between the expression of

\section{References}

1. Folkman J, Merler E, Abernathy C, Williams G. Isolation of a tumor factor responsible for angiogenesis. J Exp Med 1971; 133: $275-288$.

2. Fox $\mathrm{SB}$, Generali $\mathrm{DG}$, Harris AL. Breast tumour angiogenesis. Breast Cancer Res 2007; 9: 216.

3. Doyle DM, Miller KD. Development of new targeted therapies for breast cancer. Breast Cancer 2008; 15: 49-56.

4. Uzzan B, Nicolas P, Cucherat M, Perret GY. Microvessel density
TIMP-2 and overall survival. In contrast, Nakopoulou et al. (31) observed a better survival in cases with positive TIMP-2 and negative MMP-2 expression, and also in cases that were positive for both factors. Other investigators also reported that changes in the balance between MMPs and TIMPs with an increase in the activity of MMPs played an important role in tumor invasion and metastasis $(22,34)$.

Currently, the angiogenic factors represent an attractive therapeutic target for a new class of anticancer drugs. Although the use of antiangiogenic drugs for the treatment of cancer has increased in recent decades in basic clinical research, their clinical benefits are relatively low (35-38). Bevacizumab (Avastin, Genentech Inc., USA), is the most investigated antiangiogenic agent $(2,39)$. Miller et al. (40) recently published a randomized phase III study comparing the use of paclitaxel associated with bevacizumab versus paclitaxel alone for the initial treatment of metastases in breast cancer. The results showed a significant increase in objective response rates (36.9 vs $21.2 \%, \mathrm{P}<0.001)$ and in disease-free survival (median, 11.8 vs 5.9 months, $\mathrm{P}<0.001$ ), both in favor of the paclitaxel/bevacizumab combination.

A better definition of the biological nature of tumors allows individual approaches based on knowledge of the molecular profile of each patient. The use of antiangiogenic agents in combination with other chemotherapeutic agents can improve the efficiency of treatment and prolong the lives of patients with metastatic disease and prevent recurrence in those with initial disease $(3,10)$.

The use of angiogenic factors to identify patient prognosis in early breast cancer is not clear. The data presented in the literature are conflicting and this will remain a problem until the mechanisms involved in carcinogenesis and metastatic invasion are better understood.

In our series of patients with primary breast cancer, a larger tumor diameter and the presence of vascular invasion were correlated with a positive SLN. Higher VEGF levels and lower TIMP-1 levels were shown to be more frequently associated with tumors of the ductal type, while higher TIMP-1 levels were found in patients with tumors with lobular type. Lower TIMP-2 levels showed also a significant correlation with younger patient age and larger tumor diameter. These results warrant further studies. as a prognostic factor in women with breast cancer: a systematic review of the literature and meta-analysis. Cancer Res 2004; 64: 2941-2955.

5. Curran S, Murray GI. Matrix metalloproteinases: molecular aspects of their roles in tumour invasion and metastasis. Eur $J$ Cancer 2000; 36: 1621-1630.

6. Duffy MJ, Maguire TM, Hill A, McDermott E, O'Higgins N. Metalloproteinases: role in breast carcinogenesis, invasion and 
metastasis. Breast Cancer Res 2000; 2: 252-257.

7. Rundhaug JE. Matrix metalloproteinases and angiogenesis. $J$ Cell Mol Med 2005; 9: 267-285.

8. Locopo N, Fanelli M, Gasparini G. Clinical significance of angiogenic factors in breast cancer. Breast Cancer Res Treat 1998; 52: 159-173.

9. Hayes DF, Miller K, Sledge G. Angiogenesis as targeted breast cancer therapy. Breast 2007; 16 (Suppl 2): S17-S19.

10. Banerjee S, Dowsett M, Ashworth A, Martin LA. Mechanisms of disease: angiogenesis and the management of breast cancer. Nat Clin Pract Oncol 2007; 4: 536-550.

11. Shinkaruk S, Bayle M, Lain G, Deleris G. Vascular endothelial cell growth factor (VEGF), an emerging target for cancer chemotherapy. Curr Med Chem Anticancer Agents 2003; 3: 95-117.

12. Bos R, Zhong H, Hanrahan CF, Mommers EC, Semenza GL, Pinedo HM, et al. Levels of hypoxia-inducible factor-1 alpha during breast carcinogenesis. J Natl Cancer Inst 2001; 93: 309314.

13. Gasparini G. Prognostic value of vascular endothelial growth factor in breast cancer. Oncologist 2000; 5 (Suppl 1): 37-44.

14. Brown PD. Matrix metalloproteinase inhibitors. Breast Cancer Res Treat 1998; 52: 125-136.

15. Barr MP, Bouchier-Hayes DJ, Harmey JJ. Vascular endothelial growth factor is an autocrine survival factor for breast tumour cells under hypoxia. Int J Oncol 2008; 32: 41-48.

16. Talvensaari-Mattila A, Paakko P, Blanco-Sequeiros $G$, Turpeenniemi-Hujanen T. Matrix metalloproteinase-2 (MMP-2) is associated with the risk for a relapse in postmenopausal patients with node-positive breast carcinoma treated with antiestrogen adjuvant therapy. Breast Cancer Res Treat 2001; 65: 55-61.

17. Hirvonen R, Talvensaari-Mattila A, Paakko P, TurpeenniemiHujanen T. Matrix metalloproteinase-2 (MMP-2) in T(1-2)N0 breast carcinoma. Breast Cancer Res Treat 2003; 77: 85-91.

18. Visse R, Nagase H. Matrix metalloproteinases and tissue inhibitors of metalloproteinases: structure, function, and biochemistry. Circ Res 2003; 92: 827-839.

19. Payne SJ, Bowen RL, Jones JL, Wells CA. Predictive markers in breast cancer - the present. Histopathology 2008; 52: 8290.

20. Elston CW, Ellis IO. Pathological prognostic factors in breast cancer. I. The value of histological grade in breast cancer: experience from a large study with long-term follow-up. Histopathology 1991; 19: 403-410.

21. Scorilas A, Karameris A, Arnogiannaki N, Ardavanis A, Bassilopoulos $\mathrm{P}$, Trangas $\mathrm{T}$, et al. Overexpression of matrix-metalloproteinase-9 in human breast cancer: a potential favourable indicator in node-negative patients. $\mathrm{Br} J$ Cancer 2001; 84: 1488-1496.

22. Jinga DC, Blidaru A, Condrea I, Ardeleanu C, Dragomir C, Szegli G, et al. MMP-9 and MMP-2 gelatinases and TIMP-1 and TIMP-2 inhibitors in breast cancer: correlations with prognostic factors. J Cell Mol Med 2006; 10: 499-510.

23. Tsakonas $\mathrm{G}$, Kosmas $\mathrm{C}$. Integration of novel targeted therapies into the systemic treatment of breast cancer - a review. J BUON 2007; 12: 319-327.

24. Garbett EA, Reed MW, Stephenson TJ, Brown NJ. Proteolysis in human breast cancer. Mol Pathol 2000; 53: 99-106.

25. Li HC, Cao DC, Liu Y, Hou YF, Wu J, Lu JS, et al. Prognostic value of matrix metalloproteinases (MMP-2 and MMP-9) in patients with lymph node-negative breast carcinoma. Breast
Cancer Res Treat 2004; 88: 75-85.

26. Pellikainen JM, Ropponen KM, Kataja VV, Kellokoski JK, Eskelinen MJ, Kosma VM. Expression of matrix metalloproteinase (MMP)-2 and MMP-9 in breast cancer with a special reference to activator protein-2, HER2, and prognosis. Clin Cancer Res 2004; 10: 7621-7628.

27. Baker EA, Stephenson TJ, Reed MW, Brown NJ. Expression of proteinases and inhibitors in human breast cancer progression and survival. Mol Pathol 2002; 55: 300-304.

28. Mylona E, Nomikos A, Magkou C, Kamberou M, Papassideri I, Keramopoulos A, et al. The clinicopathological and prognostic significance of membrane type 1 matrix metalloproteinase (MT1-MMP) and MMP-9 according to their localization in invasive breast carcinoma. Histopathology 2007; 50: 338-347.

29. Choi WW, Lewis MM, Lawson D, Yin-Goen Q, Birdsong GG, Cotsonis GA, et al. Angiogenic and lymphangiogenic microvessel density in breast carcinoma: correlation with clinicopathologic parameters and VEGF-family gene expression. Mod Pathol 2005; 18: 143-152.

30. Hao L, Zhang C, Qu Y, Wang L, Luo Y, Jin M, et al. Recombination of CXCR4, VEGF, and MMP-9 predicting lymph node metastasis in human breast cancer. Cancer Lett 2007; 253: 34-42.

31. Nakopoulou L, Tsirmpa I, Alexandrou P, Louvrou A, Ampela C, Markaki S, et al. MMP-2 protein in invasive breast cancer and the impact of MMP-2/TIMP-2 phenotype on overall survival. Breast Cancer Res Treat 2003; 77: 145-155.

32. Kim HJ, Park Cl, Park BW, Lee HD, Jung WH. Expression of MT-1 MMP, MMP2, MMP9 and TIMP2 mRNAs in ductal carcinoma in situ and invasive ductal carcinoma of the breast. Yonsei Med J 2006; 47: 333-342.

33. Nakopoulou L, Giannopoulou I, Lazaris A, Alexandrou P, Tsirmpa I, Markaki S, et al. The favorable prognostic impact of tissue inhibitor of matrix metalloproteinases-1 protein overexpression in breast cancer cells. APMIS 2003; 111: 1027-1036.

34. Têtu B, Brisson J, Wang CS, Lapointe H, Beaudry G, Blanchette C, et al. The influence of MMP-14, TIMP-2 and MMP-2 expression on breast cancer prognosis. Breast Cancer Res 2006; 8: R28.

35. Sledge GW Jr. Implications of the new biology for therapy in breast cancer. Semin Oncol 1996; 23: 76-81.

36. Kerbel RS. Tumor angiogenesis. NEngl J Med 2008; 358: 20392049.

37. Hidalgo M, Eckhardt SG. Development of matrix metalloproteinase inhibitors in cancer therapy. J Natl Cancer Inst 2001; 93: 178-193.

38. Sparano JA, Bernardo P, Stephenson P, Gradishar WJ, Ingle JN, Zucker S, et al. Randomized phase III trial of marimastat versus placebo in patients with metastatic breast cancer who have responding or stable disease after first-line chemotherapy: Eastern Cooperative Oncology Group trial E2196. J Clin Oncol 2004; 22: 4683-4690.

39. Wedam SB, Low JA, Yang SX, Chow CK, Choyke P, Danforth $D$, et al. Antiangiogenic and antitumor effects of bevacizumab in patients with inflammatory and locally advanced breast cancer. J Clin Oncol 2006; 24: 769-777.

40. Miller K, Wang M, Gralow J, Dickler M, Cobleigh M, Perez EA, et al. Paclitaxel plus bevacizumab versus paclitaxel alone for metastatic breast cancer. N Engl J Med 2007; 357: 26662676. 